\title{
Molecular targets for therapeutic interventions in human papillomavirus-related cancers (Review)
}

\author{
ALAN SAITO RAMALHO $^{1}$, ANSELMO DANTAS LOPES ${ }^{1}$, ALEYLOVE TALANS ${ }^{1}$, \\ BRUNO YOSHIHIRO PARLATO SAKIYAMA ${ }^{1}$, GUILHERME LUIZ STELKO PEREIRA ${ }^{2}$, \\ PAULO MARCELO HOFF ${ }^{1,2}$ and GILBERTO DE CASTRO $\mathrm{Jr}^{2}$ \\ ${ }^{1}$ University of São Paulo, Medical School, Av. Dr. Arnaldo 455, São Paulo, SP 01246903; ${ }^{2}$ Clinical Oncology, \\ Instituto do Câncer do Estado de São Paulo, Av. Dr. Arnaldo 251, São Paulo, SP 01246000, Brazil
}

Received September 8, 2010; Accepted September 29, 2010

DOI: 10.3892/or_00001001

\begin{abstract}
The infection by the human papillomavirus (HPV) is the origin of several cancers around the world. In some areas of Brazil, cervical carcinoma is still the cancer with the highest incidence among women. After epithelial cell transformation by HPV, several molecular events are observed, resulting in the malignant phenotype. In this review we discuss potential molecular targets for therapeutic interventions in human HPV-related carcinomas, with emphasis on cervical cancer, based on the alterations observed in the signaling transduction pathways caused by HPV infection. With a special attention to tyrosine kinase receptors, and other kinases involved in signal transduction and angiogenesis, these pathological alterations are evaluated as novel targets for anticancer therapies in HPV-related carcinomas.
\end{abstract}

\section{Contents}

1. Introduction

2. Carcinogenesis in HPV-related cancers

3. Molecular targets for therapeutic interventions in HPVrelated cancers

4. Conclusion

\section{Introduction}

Human papillomaviruses (HPVs) are DNA-viruses capable of infecting basal epithelial cells. The relationship between HPV and cancer is well established and the infection by this virus is required for cervical cancer development, which is

Correspondence to: Dr Gilberto de Castro Jr, Instituto do Câncer do Estado de São Paulo - ICESP, Av. Dr. Arnaldo 251, 5th floor, São Paulo, SP, Brazil

E-mail: gilberto.castro@usp.br

Key words: human papillomaviruses, cervical cancer, tyrosine kinase receptors, mammalian target of rapamycin, molecular targeted therapies responsible for the death of around 274,000 women per year worldwide $(1,2)$. Besides cervical cancer, HPV is also associated with penile, vulvar, anal and oropharyngeal squamous cell carcinomas $(3,4)$. The infection by high-risk oncogenic HPVs is estimated to lead to around $100 \%$ of cervical cancers, $90 \%$ of anal cancers, $40 \%$ of vulvar and penile cancers and, at least, $12 \%$ of oropharyngeal cancers reaching almost $60 \%$ of tonsillar cancers $(2,5,6)$. Particularly in Brazil, although screening of precursor lesions and invasive carcinoma by Pap smear is regularly performed, cervical cancer still represents the cancer with highest incidence (22 cases/100,000 inhabitants) in the Northern region of the country (7).

Since the 1970s, when Harald Zur Hausen, awarded with the Nobel Prize in Physiology or Medicine in 2008, focused his research on the relationship between HPVs and cancer, more than one hundred of HPV types have been described and their genetic sequences identified $(8,9)$. This knowledge led to the classification of different viral types and their denomination by cardinal numbers according to the organization of nucleotide sequences of viral genome.

The prevalence of HPV infection is highly variable in different populations, oscillating between $1.4 \%$ and $25.6 \%$ (10). The highest prevalence is found in women under the age of 25 and there is a progressive decline after this age (11). Currently, HPV infection is the most frequent sexually transmitted disease $(12,13)$. In 2003, mortality-related productivity costs of HPV-associated cancers in the USA reached US\$ 3.7 billions, showing the socio-economic challenge of tumors associated with HPV infections (14).

In this review, the authors discuss the molecular targets to be studied with therapeutic purposes in malignant tumors associated with HPV infections, particularly in cervical carcinoma, based on the alterations in signaling transduction pathways caused by this virus in infected cells (Table I), and also taking into account the recent knowledge on HPV carcinogenic mechanisms and the abundance of information on molecular targeted therapies in clinical development.

A data search was performed using Pubmed database for English-language studies from January, 2000, to May, 2010. We searched using the terms 'molecular targeted therapy', 'signaling pathway', 'HER', 'EGFR', 'c-MET', 'PI3K', 'Akt', 'mTOR', 'PTEN', 'MAPK', 'Ras', 'Raf', 'MEK', 'VEGF', 
'HIF', 'everolimus', 'temsirolimus', 'cetuximab', linked to 'E6', 'E7', 'tumor', 'cervical cancer', 'anal cancer', 'penile cancer', and/or 'oropharynx cancer'. The list of publications was narrowed to relevant studies of the studied period. References of this list of publications were reviewed, in order to extract other relevant articles. Personal papers of authors were also used in this review.

\section{Carcinogenesis in HPV-related cancers}

HPV infection can be caused by different types of HPV. They can be classified into high-oncogenic risk, which are mainly found in cervical cancer and in high-grade squamous intraepithelial lesions, and in low-oncogenic risk, usually found in genital warts. Co-infection by high- and low-risk types is a common event (15).

HPV types 16 and 18 are the most associated with cervical carcinoma, present in more than $70 \%$ of the cases, despite some geographic variation $(4,8,10)$. Indeed, the following types have also been classified as carcinogenic: 31 , 33, 35, 39, 45, 51, $52,56,58,59$ and 66 (4).

Usually, cervix infection by HPV is a transitory event, commonly in young woman when sexual life is initialized, and no HPV is detected after 36 months in more than $90 \%$ of cases (12). Inside the host cell, HPV DNA can assume two different forms: episomal and integrated. When the viral DNA is in the cell nucleus, but not bound to the host DNA, it is in the episomal form. As soon as it binds to the host DNA, it is considered integrated, which is found in high-grade lesions and invasive carcinoma $(15,16)$.

There are three viral genes related to epithelial proliferation of infected cells. E5 product seems to be important in the early phases of infection. During the viral integration, E6 and E7 command carcinogenesis. E6 and E7 products induce increased cell proliferation, causing epithelial atypical features and, ultimately, the malignant phenotype. E6 and E7 proteins are the only viral products conserved and continuously expressed in cervical cancer cells, indicating their importance in cancer progression. Their main function is to enhance the degradation of tumor suppressor proteins $\mathrm{p} 53$ and the product of the retinoblastoma gene (pRb), respectively $(17,18), \mathrm{pRb}$ degradation by $\mathrm{E} 7$ prevents it from binding to the transcription factor E2F that regulates the expression of genes involved in progression to the S-phase of cell cycle, such as cyclin D and $C D K 2$ genes. As pRb normally acts as a negative regulator of p16 ${ }^{\mathrm{INK} 4 \mathrm{~A}}$ (p16) expression, a tumor suppressor gene located on chromosome $9 \mathrm{p} 21$, the inactivation of $\mathrm{pRb}$ results in overexpression of $\mathrm{p} 16$ protein. The $\mathrm{p} 53$ mediated apoptosis is blocked by E6. Thus, E6 and E7 act synergistically to maintain the malignant phenotype $(17,19)$. It is well established that HPV products can affect tumor cell characteristics, including growth regulators independence, resistance to apoptosis, immune escape and angiogenesis, as shown in Fig. 1 (19-23).

\section{Molecular targets for therapeutic interventions in HPV- related cancers}

\section{Receptor kinases}

Human epidermal growth factor receptor family. The human epidermal growth factor receptor (HER) family members include four receptors: EGFR (epidermal growth factor receptor or HER-1), HER-2/neu, HER-3 and HER-4. These receptors are located in the cell surface and have three domains: extracellular, transmembrane and intracellular, and all, except HER3, contain an intracellular tyrosine kinase domain. The activation of these receptors by specific ligands leads to dimerization with auto-phosphorylation and subsequent activation of the tyrosine kinase, which induces a cascade of downstream signaling through several pathways, such as PI3K/Akt/mTOR, Ras/Raf/MAPK and JAK/STAT, resulting in cellular proliferation, diminished apoptosis, increased motility and adhesion properties, and increased DNA repair (Fig. 1).

The amplification of the gene encoding EGFR is strongly associated with overexpression of $\mathrm{p} 16$, as shown by Kim et al (24). In this study, $71.2 \%$ of patients with tonsillar cancer expressed p16 while no expression occurred in chronic follicular tonsillitis.

Since the overexpression of EGFR has been identified as an independent predictor of poor prognosis in cervical carcinoma $(25,26)$, EGFR appears to be an interesting molecular target in these tumors. In the study by Kurtz et al (27), 44 patients with advanced cervical carcinoma were treated with cetuximab, a chimeric anti-EGFR monoclonal antibody, in combination with cisplatin and topotecan. Unfortunately, this study was stopped early due to severe bone marrow toxicity and life-threading infections.

In a phase II study presented by Ferreira et al (28), 23 patients with squamous cell cervical cancer were treated with chemoradiation (45 Gy, followed by 4 applications of brachytherapy $600 \mathrm{cGy}$ concurrent to cisplatin $40 \mathrm{mg} / \mathrm{m}^{2} /$ week, 5 weeks), associated with erlotinib (150 mg/d orally), a small molecule EGFR tyrosine kinase inhibitor. There was an impressive $91 \%$ rate of complete response (28). However, another recent phase II trial in patients with advanced disease showed that monotherapy with erlotinib was ineffective, with no objective response in 25 studied patients (29).

Hepatocyte growth factor and c-Met axis. c-Met is a membrane-spanning receptor involved in several biological activities including motility, proliferation, survival, invasion and morphogenesis (30). Hepatocyte growth factor (HGF) is the only known ligand of c-Met (31). The HGF/c-Met axis is implicated in a wide variety of epithelial, mesenchymal, and hematological malignancies (Fig. 1).

Walker et al (32) reported that HGF and c-Met levels were significantly correlated with the severity of intraepithelial lesions infected with oncogenic HPVs. The correlation between oncogenic HPV infection and overexpression of the complex HGF/c-Met in cervical lesions of squamous epithelia is a strong indication that HGF/c-Met axis may be a potential cancer target for future therapeutic interventions in these cancers. There are currently several HGF/c-Met inhibitors under clinical evaluation, including the monoclonal antibody AMG102, and some small-molecule inhibitors (XL880, PF02341066).

Insulin-like growth factor receptor. Similar to the EGFR pathway, the insulin-like growth factor receptor (IGFR) signaling system comprises multiple circulating ligands, such 
Table I. Signal transduction pathways, molecular targets and drugs in clinical development for therapeutic interventions in HPV-related cancers.

\begin{tabular}{lll}
\hline $\begin{array}{l}\text { Receptors and signaling transduction } \\
\text { pathways }\end{array}$ & Molecular targets & \multicolumn{1}{c}{ Drugs in clinical development } \\
\hline $\begin{array}{l}\text { Receptor kinases } \\
\text { EGFR pathway }\end{array}$ & EGFR & Cetuximab, panitumumab, nimotuzumab, necitumumab \\
HGF pathway & c-Met & AMG102, XL880, PF02341066 \\
IGFR pathway & IGF-1R & CP721871, BIIB022, XL228, OSI-906 \\
Intracellular signaling kinases & & \\
PI3K/Akt/mTOR & PI3K & PI103, BGT226 \\
& Akt & Perifosine, GSK690693 \\
& mTOR & Temsirolimus, everolimus, ridaforolimus \\
Ras/Raf/MAPK & Ras & Tipifamib, lonafamib \\
& Raf & Sorafenib, PLX4032 \\
& MAPK & AZD6244, XL518 \\
Angiogenesis & VEGF, VEGFR & Bevacizumab, sunitinib, AZD2171 \\
& HIF-1 $\alpha$ & Adaphostin \\
\hline
\end{tabular}

EGFR, epidermal growth factor receptor; HGF, hepatocyte growth factor; HIF-1 $\alpha$, subunit $\alpha$ hypoxia-inducible factor; IGFR, insulin-like growth factor receptor; IGF-1R, type I insulin-like growth factor receptor; PI3K, phosphoinositide 3' kinase; MAPK, mitogen-activated protein kinase; mTOR, mammalian target of rapamycin; VEGF, vascular endothelial growth factor; VEGFR, vascular endothelial growth factor receptor.

as IGF-1, IGF-2 and insulin, interacting with membrane-bound receptors, such as type 1 IGF receptor (IGF-1R) and insulin receptor (IR). The IGF-1R undergoes conformational changes and phosphorylation upon ligand binding and recruits insulinreceptor substrates and/or Scr homology 2 domain-containing proteins. The mitogenic, proliferative and/or anti-apoptotic signals are then transmitted downstream through MAPK and PI3K/Akt/mTOR pathways (Fig. 1) (33).

In the physiological state, IGF-1R plays an important role in fetal development and linear growth of many organs, whereas insulin/IR interaction regulates carbohydrates and lipid metabolism, among other functions. However IGF-1R was implicated in the development and maintenance of malignant phenotype, and interruption of IGF-1R signaling inhibited cancer cell growth and motility both in vitro and in vivo (34).

Aberrant activation of the IGF-1/IGF-1R pathway was also associated with worse prognosis in many malignant neoplasms, including multiple myeloma, prostate cancer, nonsmall cell lung cancer and renal cell cancer. Aside from the IGF-1R, abnormally activated IR by insulin or IGF-2 stimulation enhances proliferation in cancer cells, thus highlighting their therapeutic value as molecular targets in cancer. However, IR inhibition may lead to type 2 diabetes mellitus and IGF-1deficient states have been associated with osteoporotic fractures and ischemic heart disease (35).

Serrano et al (36) showed that the expression of IGF receptors are different between HPV positive (Siha) and negative (C33a) human cervical cancer cell lines. Siha cells express the three receptors (IGF-1R, IR-A, IR-B, and hybrids) whereas C33a mainly express IR-A. They found that both IGF-1 and IGF-2 can stimulate IR and IGF-1R autophosphorylation while insulin only stimulates IR autophosphorylation at physiological concentrations. In the $\mathrm{C} 33$ a cell line the three ligands stimulate the phosphorylation of IR, but lower concentrations of IGF-2 are needed in comparison to IGF-1. This result is consistent with the predominance of IR-A in C33a and the higher affinity of IR-A for IGF-2 than IGF-1. In agreement with the presence of receptors in $\mathrm{C} 33 \mathrm{a}$ and SiHa cells, it was observed that activation of PI3K and MAPK pathways occurs in response to stimuli with all three ligands. It was also described a higher basal Akt phosphorylation in C33a cells that mainly express IR-A and have PTEN (phosphatase and tensin homolog) mutations, which was increased by treatment with IGF-I and IGF-II, but not with insulin, in comparison to $\mathrm{SiHa}$ cell line. Thus, studies of therapeutic agents that interact with IGFR in the treatment of cervical cancer should consider the different patterns of receptor distribution of the IGFR pathway. HPV-positive tumors express significantly more IGF-1R, and IGF-1 has an important role in the survival of these cells, which makes the complex IGF-1/IGF-1R an interesting target in the treatment of cervical cancer.

There are several IGF-1R-targeting agents in clinical development, including monoclonal antibodies, such as $\mathrm{CP}$ 721871 and BIIB022, and small molecule inhibitors, such as XL-228 and OSI-906, but none are approved for current clinical use in oncology.

Intracellular signaling kinases.

PI3K/Akt/mTOR pathway. The phosphatidylinositol 3-kinase (PI3K)/Akt/mTOR (mammalian target of rapamycin) pathway acts as a cell sensor for nutrients and growth factors, integrating signals from multiple receptors to regulate cellular growth and metabolism (37). Although this pathway is often described as linear and vertical, its regulation is complex, 


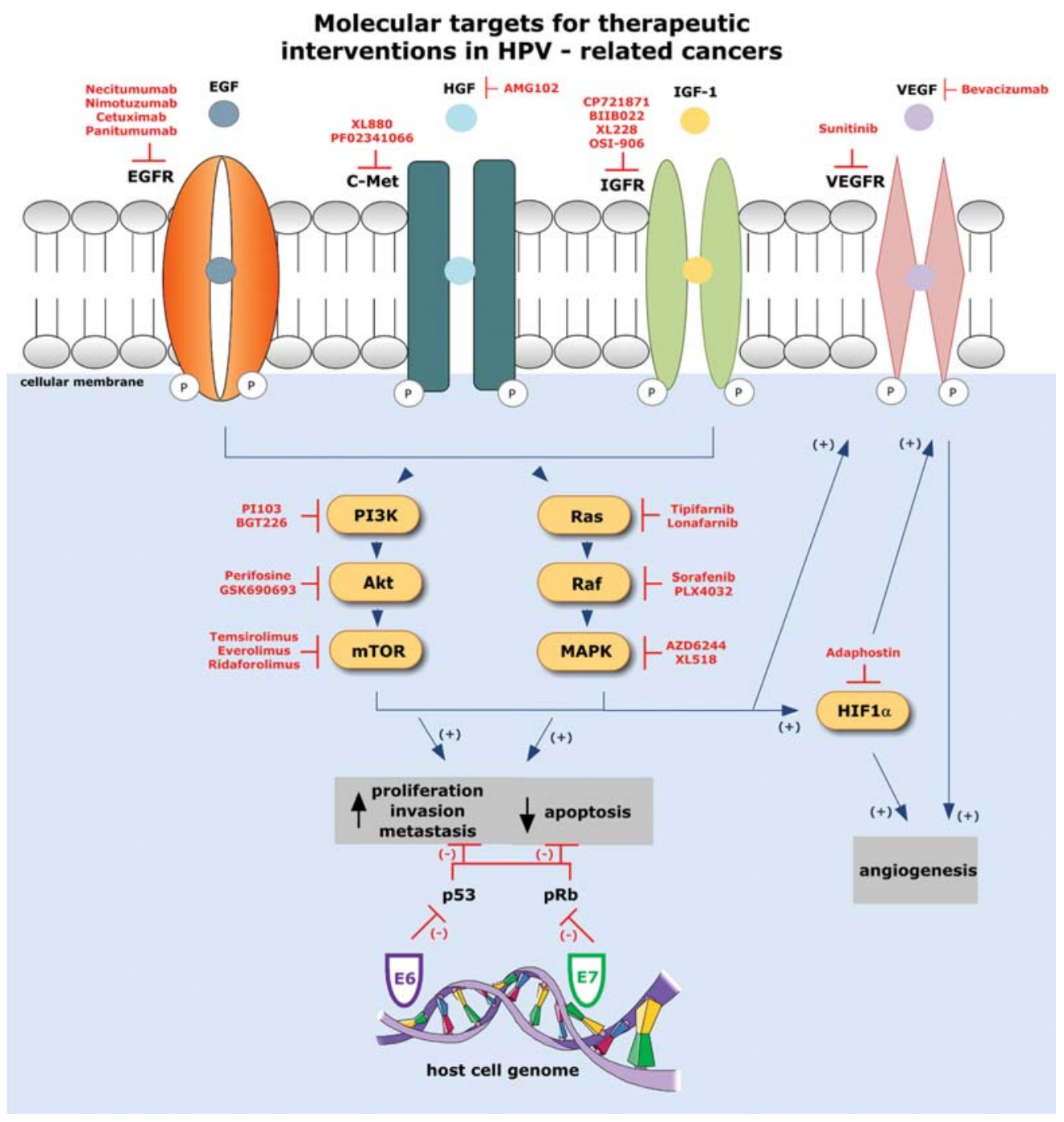

Figure 1. Molecular targets for therapeutic interventions in HPV-related cancers. Targeted therapies are indicated in red. c-MET, HGF receptor; E6, HPV early protein 6; E7, HPV early protein 7; EGF, epidermal growth factor; EGFR, epidermal growth factor receptor; HGF, hepatocyte growth factor; HIF-1 $\alpha$, subunit $\alpha$ of the hypoxia-inducible factor 1; IGF-1, type I insulin-like growth factor; IGFR, insulin-like growth factor receptor; MAPK, mitogen-activated protein kinase; mTOR, mammalian target of rapamycin; p53, protein 53; PI3K, phosphoinositide 3'-kinase; pRb, retinoblastoma protein; VEGF, vascular endothelial growth factor; VEGFR, vascular endothelial growth factor receptor.

with internal feedbacks and crosstalks with other intracellular signaling pathways, such as MAPK.

PI3K is a lipid kinase that generates the 3'-phosphoinositides (PIP3) in the cell membrane when activated by receptor kinases. This leads to the recruitment of phosphoinositidedependent kinase (PDK1) and Akt to the cell membrane. The formation of PIP3 is negatively regulated by PTEN. Akt is activated by multiple enzymes, including PDK1, mTORC 2 and IRS-1, leading to inhibition of protein 2 tuberous sclerosis complex (TSC2). Inhibition of TSC leads to mTOR-mediated activation of 4EBP1 and p70S6K, which in turn regulate cellular mechanisms of gene transcription and translation (Fig. 1) (37).

The PI3K/Akt/mTOR pathway is a promising target for molecular therapies for several reasons. The kinases of this pathway are overactivated in a variety of solid tumors types, as a result of loss of PTEN function, amplification of Akt, mutations of TSC and/or constitutional activation of upstream kinases (38).

Fausch et al (39) showed that the activation of the PI3K in Langerhans cells stimulated by HPV virus-like particles was associated with reduced immune response, due to repression of transcription of important genes related to immune function. When PI3K activation was suppressed, the Langerhans cells were able again to trigger an efficient immune response.

Among the compounds targeting the PI3K/Akt/mTOR pathway, mTOR inhibitors are furthest along in development. The mTOR protein is a serine-threonine kinase discovered in the 1990s when the mechanism of action of rapamycin was investigated. Rapamycin (or sirolimus) is a macrolide first discovered as a product of Streptomyces hygroscopicus, a bacterium isolated in a soil sample from Easter Island, and has been widely used as immunosuppressive agent in organ transplantations (37).

Activation of $\mathrm{PI} 3 \mathrm{~K} / \mathrm{Akt} / \mathrm{mTOR}$ pathway was associated with a worse prognosis and chemoresistance in cervical cancer. Faried et al (40) found that the survival of patients with mTOR-negative cervical adenocarcinomas was significantly higher than those of mTOR-positive tumors, suggesting that the expression of mTOR is an independent marker of worse prognosis. 
The inhibition of mTOR increases the response to cytotoxic chemotherapy. Treatment of CaSki cervix cells with rapamycin increased the sensitivity to paclitaxel and inhibited several proteins involved in cell survival, suggesting a synergistic role between rapamycin and cytotoxic agents in this model (41). Brown et al (42) showed that in most cases of oropharyngeal carcinoma, there is an increased constitutional activation of the mTOR pathway. It was also observed that rapamycin caused a reduction in the expression of $\mathrm{p}$-mTOR (phosphorylated Ser-2448) in these tumors, which inhibited cell growth in a dose-response pattern.

Akt is a serine-threonine kinase that regulates mTORC1 and it is implicated in carcinogenesis in several malignancies. Akt is equally attractive as mTOR for molecular targeting in therapeutics due to its participation in important cellular functions, including cell cycle progression, gene transcription and translation, apoptosis and cellular metabolism. Bertelsen et al (43) observed that in cervical carcinogenesis there was an increased activation of Akt due to PI3KCA gene amplification, raising the intracellular levels of PI3K. The mechanism of Akt activation by amplification of PIK3CA was also demonstrated in anal squamous cell carcinoma. Furthermore, in squamous cell carcinoma of head and neck, PIK3CA was found overexpressed in HPV-positive lesions when compared to those HPV-negative.

It was shown that E7 protein is able to modulate the cytoplasmic localization of $\mathrm{p} 27$, a regulator of cell motility, which acts by inhibiting the activation of the cytoskeleton organizer RhoA. Modulation by E7 on p27 occurs through the activation of the PI3K/Akt pathway. It follows, therefore, that mobility and cell migration can also be dependent on activated Akt (44). Moreover, Menges et al (45) showed that the expression of E7 protein of HPV-16 in cervical keratinocytes cause inhibition of cell differentiation and proliferation, and increased activity of Akt.

The increased activity of Akt is dependent on the ability of E7 to bind with and to inactivate the $\mathrm{Rb}$ protein. The knockout of $R b$ with shRNAs, small hairpin RNAs capable of blocking mRNA by RNA-induced silencing complex, was capable of increasing Akt phosphorylation in many cell cultures. As HPV is strongly associated as a cause of cervical cancer, it was also evaluated whether the expression of Akt was increased in cervical biopsies, which in fact has been confirmed, as there was a higher expression of Akt in neoplastic in comparison to normal tissue, strongly correlated with the expression of $E 7$ (45).

Another oncoprotein involved in this process is E6, which modulates the activity of PTEN, leading to a decrease of its activity. Since PTEN is a negative regulator of Akt, E6 expression results in the stimulation of Akt pathway. ContrerasParedes et al (46) showed that E6 was also able to activate PI3K and MAPKs, particularly ERK1 and ERK2. Thus, the inhibition of Akt signaling, either directly or by interfering with regulators of PI3K pathway is a potential treatment strategy in cervical cancer.

Given the pivotal role of Akt in the cellular homeostasis, its inhibition leads to more frequent severe adverse effects than the inhibition of mTOR, and it is one of the possible reasons for the disappointing development of Akt inhibitors (47).
Ras/Raf/MAPK pathway. Mitogen-activated protein kinase (MAPK) pathway is referred to be as a major connector between extracellular and intracellular stimuli such as cytokines, growth factors, oncogenes and cellular responses related to adhesion, mobility and malignant transformation (48). Ras is a GTPase protein which transmits activating signals from growth factors, cytokines, and oncogenes to Raf and then to MAPK. Following, MAPK phosphorylates and activates the extracellular signal-regulated kinase (ERK) (Fig. 1).

H-Ras, K-Ras and N-Ras genes, in humans, have been localized to chromosome 11, 12 and 1, respectively, and their activations by point mutations have been suggested to play an important role in the carcinogenesis process $(49,50)$. It is estimated that activating Ras mutations are found in more than $20 \%$ of human cancers (51).

The exact mechanisms linking overexpression of Ras genes and cervical carcinogenesis remain to be elucidated, however, a cooperative effect between Ras and E6/E7 genes in cellular transformation is considered $(52,53)$. The activation of ERK1 and ERK2 as a consequence of E6/E7 transcription has been already observed (46).

Regarding the association between high-risk HPVs and genetic alterations in $\mathrm{H}$-Ras, there is evidence of a more aggressive tumor behavior in patients whose tumors harbor $\mathrm{H}$-Ras mutations, as an early predictor of faster tumor progression or higher risk of invasion (54). Actually, cells with H-Ras mutations were reported to present higher proliferation rate when infected by HPV, leading to reduced duration of G1 phase of cell cycle, dependent on MAPK and/or PI3K/Akt/ mTOR activation (55). As a consequence, poorly differentiated tumors were observed in patients with cervical squamous cell carcinoma carrying H-Ras mutations $(56,57)$.

Besides its prognostic value, the expression of the oncogenic Ras-family proteins seems to be predictive of resistance of cancer cells to radiation therapy. It has been reported that inhibition of H-Ras, by treatment of a transformed embryonic rat fibroblast cell line with a farnesyltransferase selective inhibitor, resulted in higher levels of apoptosis after radiation $(58,59)$. Post-translational modifications, such as farnesylation, are required for the membrane localization and activation of Ras. This information has led to an interest in developing farnesyltransferase inhibitors (FTIs) which could prevent the proper functioning of Ras. Some examples are tipifarnib and lonafarnib, which unfortunately did not show relevant clinical activity in phase II and III studies $(60,61)$.

The RASSF $1 A$ gene, identified at chromosome region 3 p21, encodes a novel Ras-binding protein. The precise function of this gene is not well known, but it is suggested that its overexpression induces apoptosis, demonstrating tumor suppressor gene properties $(62,63)$. RASSF 1A is inactivated by DNA promoter hypermethylation in several human solid tumors, including cervical cancers, being probably the most commonly inactivated gene thus far reported in human cancer $(64,65)$.

The prevalence of RASSF $1 A$ promoter methylation in cervical adenocarcinoma is around $20 \%$, and there is an inverse correlation between RASSF $1 A$ inactivation and the presence of HPV-transforming gene products $(65,66)$. It may suggest that RASSF 1A promoter methylation plays an important 
role in the development of cervical adenocarcinoma, independently of HPV infection status. The epigenetic alteration that interferes in Ras pathway may have therapeutic implications, as it could explain some possible differences in therapeutic responses between cervical adeno- and squamous cell carcinomas.

Angiogenesis. Angiogenesis describes the formation of new vessels from existing vasculature. It is vital in some physiological process, such as wound healing, menstrual cycle and embryogenesis, but it is dysfunctional in malignant tumors $(67,68)$. In hypoxic conditions, cells produce a large number of cytokines and growth factors that induce proliferation, migration and formation of new vessels by endothelial cells (69).

Vascular endothelial growth factors and receptors (VEGF, VEGFR) are now well validated targets in cancer therapy $(70,71)$. Small molecules that inhibit the tyrosine kinase domain of VEGFRs, like sunitinib and sorafenib, as well as the monoclonal antibody bevacizumab, directed to circulating VEGF, are now used in the daily clinical practice in oncology.

Hypoxia-inducible factors (HIF)-1 and HIF-2 are recognized as important mediators involved in tumor angiogenesis and metabolism (72). HIF-1 $\alpha$ subunit is rapidly degraded by the ubiquitin-proteasome system under normal oxygen conditions. Nonetheless, it is upregulated in a hypoxic tumor microenvironment, resulting in accumulation of HIF-1 $\alpha(69,73)$. Besides, aberrant HIF- $1 \alpha$ expression can be observed through MAPK or PI3k/Akt/mTOR stimulation, activation of oncogenes, or loss of tumor suppressor genes such as VHL, p53 and PTEN, which can lead to tumor progression, making HIF a rational target for anticancer therapies (Fig. 1) (69).

Angiogenesis is an early event in the progression of HPVrelated cervical lesions (74). A strong association between higher VEGF expression in cervical cancer and worse prognosis has been observed $(75,76)$.

The expression of HPV genes can contribute to production of angiogenic factors. Among the most studied pro-angiogenic factors, VEGF is consistently overexpressed in cervical neoplastic lesions (77). Overexpression of E6 is associated to higher production of VEGF in cervical cancer $(22,77)$. Studies using transgenic mice as well as samples of human cervical tissue suggest that both E6 and E7, besides hypoxia, can stimulate VEGF production $(78,79)$. López-Ocejo et al (77) have demonstrated that E6 positive cervical carcinoma cells expressed VEGF mRNA levels two to three times higher than E6 negative cells. Moreover, reduction in VEGF expression was able to produce suppressive effects over angiogenesis and tumor growth in vivo. In HPV-16 positive cells, E6 oncoprotein can contribute to amplification of epigenetic alterations that increase the expression of VEGF.

In HPV-16 positive cells, expression of pro-angiogenic molecules, such as bFGF, IL-8, TNF $\alpha$, TGFß and VEGF was higher as compared to control keratinocytes. In contrast, expression of the anti-angiogenic factors trombospondin (TSP)-1 and 2 were decreased in cells infected by HPV. It demonstrates that cervical cancer expression of HPV-16 integrated genes is able to contribute to a pro-angiogenic phenotype (referred as angiogenic switch) that might support tumor growth and invasion $(22,74,80)$.
Bevacizumab is a monoclonal antibody that neutralizes circulating VEGF, blocking its action. Wrigth et al (81) described the first use of bevacizumab in recurrent cervical cancer. In six patients, bevacizumab was combined to cytotoxic chemotherapy (5-fluorouracil or capecitabine) and clinical benefit (any response or disease stabilization) was verified in $67 \%$ of patients. Although the number of patients was small, the benefits and safety of this therapy suggested that VEGF is a potential molecular target in recurrent cervical cancer. In the phase II study published by Monk et al (76), bevacizumab was evaluated in 46 patients with recurrent cervical cancer. Patients received bevacizumab $15 \mathrm{mg} / \mathrm{kg}$ intravenously every 21 days until disease progression or severe toxicity. Of these patients, $23.9 \%$ had at least six months of progression-free survival and $10.7 \%$ had, at least, partial response. Median progression-free and overall survival were 3.4 and 7.3 months, respectively. Treatment-related toxicity was high: eight events of hematologic grade 3 toxicity were observed, five patients had deep venous thrombosis, one patient presented grade 4 vaginal bleeding, one had a grade 4 urinary fistula and one patient died as a consequence of infection.

Besides VEGF, HIF- $1 \alpha$ is commonly overexpressed in cervical cancer, as a consequence of HPV genomic integration, and it is related to a diminished response to radiation therapy and unfavorable prognosis (82). Besides, HIF-1 $\alpha$ overexpression occurs not only in tumor cells, but also in pre-malignant lesions (83). Both E6 and E7 act independently by interfering in HIF- $1 \alpha$ production.

Therefore, HPV E6 and E7 oncoproteins can contribute to cervical cancer progression not only by deregulating the cell cycle, but also by promoting angiogenesis as well. VEGF and its receptors, besides HIF- $1 \alpha$, are promising targets to be explored as therapeutic interventions in these HPV-related cancers.

\section{Conclusion}

Advancing the knowledge of the molecular biology of HPVrelated tumors allowed to identify molecular targets, such as those here described, as growth factor receptors, signaling transduction pathways and angiogenesis. Molecular targeted drugs that are already in use in the daily practice, or in different stages of clinical development, may inhibit tumor progression and increase apoptosis, resulting in tumor response or stabilization. Due to a more rational mechanism of action, the toxicity profile and effectiveness are different from those observed with the classic cytotoxic chemotherapy. Some of these new drugs have enormous therapeutic potential for clinical applicability in the treatment of these HPV-related cancers, reducing morbidity and mortality and improving patients' quality of life. The development of these drugs through clinical studies with appropriate design and procedures, preferably with exploratory translational aspects, should be encouraged.

One aspect that must be stressed is how to best assess the HPV positivity status. Currently, p16 immunohistochemistry, in situ hybridization and E6 messenger RNA RT-PCR are being investigated and p16 immunohistochemical expression appears to be a reliable surrogate marker for relevant HPV infection $(84,85)$. 


\section{References}

1. Walboomers JM, Jacobs MV, Manos MM, et al: Human papillomavirus is a necessary cause of invasive cervical cancer worldwide. J Pathol 189: 12-19, 1999.

2. Parkin DM and Bray F: Chapter 2: the burden of HPV-related cancers. Vaccine 24: 11-25, 2006.

3. Cutts FT, Franceschi S, Goldie S, et al: Human papillomavirus and HPV vaccines: a review. Bull World Health Organ 85: 719-726, 2007

4. International Agency for Research on Cancer-IARC monograph on the evaluation of carcinogenic risks to humans. Human papillomaviruses. IARC monograph: 90, 2005.

5. Frisch M and Goodman MT: Human papillomavirus-associated carcinomas in Hawaii and the mainland U.S. Cancer 88: 1464-1469, 2000.

6. Syrjänen S: HPV infections and tonsillar carcinoma. J Clin Pathol 57: 449-455, 2004.

7. Brasil. Ministério da Saúde. Instituto Nacional de Câncer-INCA Estimativas de incidência e mortalidade por câncer. Rio de Janeiro, 2008. Available at http://www.inca.gov.br/estimativa/ 2008/versaofinal.pdf. Accessed in 16 Jul 2009.

8. Muñoz N, Bosch FX, de Sanjosé S, et al: Epidemiologic classification of human papillomavirus types associated with cervical cancer. N Engl J Med 348: 518-527, 2003.

9. zur Hausen H: Papillomaviruses causing cancer: evasion from host-cell control in early events in carcinogenesis. J Natl Cancer Inst 92: 690-698, 2000.

10. Clifford GM, Gallus S, Herrero R, et al: Worldwide distribution of human papillomavirus types in cytologically normal women in the International Agency for Research on Cancer HPV prevalence surveys: a pooled analysis. Lancet 366: 991-998, 2005 .

11. de Sanjose S, Almirall R, Lloveras B, et al: Cervical human papillomavirus infection in the female population in Barcelona, Spain. Sex Transm Dis 30: 788-793, 2003.

12. Franco EL, Villa LL, Sobrinho JP, et al: Epidemiology of acquisition and clearance of cervical human papillomavirus infection in women from a high-risk area for cervical cancer. J Infect Dis 180: 1415-1423, 1999.

13. Trottier $\mathrm{H}$ and Franco EL: The epidemiology of genital human papillomavirus infection. Vaccine 24: 1-15, 2006

14. Ekwueme DU, Chesson HW, Zhang KB and Balamurugan A: Years of potential life lost and productivity costs because of cancer mortality and for specific cancer sites where human papillomavirus may be a risk factor for carcinogenesis-United States, 2003. Cancer 113: 2936-2945, 2008.

15. Myers ER, McCrory DC, Nanda K, Bastian L and Matchar DB: Mathematical model for the natural history of human papillomavirus infection and cervical carcinogenesis. Am J Epidemiol 151: 1158-1171, 2000

16. Howley PM and Lowy DR: Papilloma viruses. In: Fields Virology. Knipe DM, Howley PM and Griffin DE (eds.) chapter 62. Lippincott, Williams \& Wilkins, Philadelphia, 5th edition, 2007

17. Munger K, Baldwin A, Edwards KM, et al: Mechanisms of human papillomavirus-induced oncogenesis. J Virol 78: 11451-11460, 2004

18. zur Hausen $\mathrm{H}$ : Papillomaviruses and cancer: from basic studies to clinical application. Nat Rev Cancer 2: 342-350, 2002.

19. zur Hausen H: Papillomaviruses in the causation of human cancers - a brief historical account. Virology 384: 260-265, 2009.

20. Hanahan D and Weinberg RA: The hallmarks of cancer. Cell 100: 5770,2000

21. O'Brien PM and Saveria-Campo M: Evasion of host immunity directed by papillomavirus-encoded proteins. Virus Res 88 : 103-117, 2002

22. Toussaint-Smith E, Donner DB and Roman A: Expression of human papillomavirus type $16 \mathrm{E} 6$ and E7 oncoproteins in primary foreskin keratinocytes is sufficient to alter the expression of angiogenic factors. Oncogene 23: 2988-2995, 2004

23. Li Y, Nichols MA, Shay JW and Xiong Y: Transcriptional repression of the D-type cyclin-dependent kinase inhibitor p16 by the retinoblastoma susceptibility gene product $\mathrm{pRb}$. Cancer Res 54: 6078-6082, 1994

24. Kim SH, Koo BS, Kang S, et al: HPV integration begins in the tonsillar crypt and leads to the alteration of p16, EGFR and c-myc during tumor formation. Int J Cancer 120: 1418-1425, 2007.
25. Kersemaekers AM, Fleuren GJ, Kenter GG, et al: Oncogene alterations in carcinoma of the uterine cervix: Overexpression of the epidermal growth factor receptor as associated with poor prognosis. Clin Cancer Res 5: 577-587, 1999.

26. Kristensen GB, Holm R, Abeler VM and Tropé CG: Evaluation of the prognostic significance of cathepsin $\mathrm{D}$ epidermal growth factor receptor and C-ERBB-2 in early cervical squamous carcinoma. Cancer 78: 433-440, 1996.

27. Kurtz JE, Hardy-Bessard AC, Deslandres M, et al: Cetuximab, topotecan and cisplatin for the treatment of advanced cervical cancer: A phase II trial. Gynecol Oncol 113: 16-20, 2009.

28. Ferreira CG, Erlich F, Carmo CC, et al: Erlotinib combined with cisplatin and radiotherapy for patients with locally advanced squamous cell cervical cancer: A phase II trial. J Clin Oncol 26: $5511,2008$.

29. Schilder RJ, Sill MW, Lee YC and Mannel R: A phase II trial of erlotinib in recurrent squamous cell carcinoma of the cervix: a Gynecologic Oncology Group Study. Int J Gynecol Cancer 19: 929-933, 2009.

30. Birchmeier C, Birchmeier W, Gherardi E and Vande Woude GF: Met, metastasis, motility and more. Nat Rev Mol Cell Biol 4: 915-925, 2003.

31. Gentile A, Trusolino L and Comoglio PM: The Met tyrosine kinase receptor in development and cancer. Cancer Metastasis Rev 27: 85-94, 2008

32. Walker F, Kermorgant S, Daraï E, Madelenat P, Cremieux AC, Hénin D, et al: Hepatocyte growth factor and c-Met in cervical intraepithelial neoplasia: overexpression of proteins associated with oncogenic human papillomavirus and human immunodeficiency virus. Clin Cancer Res 9: 273-284, 2003.

33. Sachdev D and Yee D: Disrupting insulin-like growth factor signaling as a potential cancer therapy. Mol Cancer Ther 6: 1-12, 2007.

34. Pollak M: Insulin-like growth factor-related signaling and cancer development. Recent Results Cancer Res 174: 49-53, 2007.

35. Sciacca L, Costantino A, Pandini G, et al: Insulin receptor activation by IGF-II in breast cancers: evidence for a new autocrine/ paracrine mechanism. Oncogene 18: 2471-2479, 1999.

36. Serrano ML, Sánchez-Gómez M, Bravo MM, Yakar S and LeRoith D: Differential expression of IGF-I and insulin receptor isoforms in HPV positive and negative human cervical cancer cell lines. Horm Metab Res 40: 661-667, 2008.

37. Sabatini DM: mTOR and cancer: insights into a complex relationship. Nat Rev Cancer 6: 729-734, 2006.

38. Granville CA, Memmott RM, Gills JJ and Dennis PA: Handicapping the race to develop inhibitors of the phosphoinositide 3-kinase/Akt/mammalian target of rapamycin pathway. Clin Cancer Res 12: 679-689, 2006.

39. Fausch SC, Fahey LM, Da Silva DM and Kast WM: Human papillomavirus can escape immune recognition through Langerhans cell phosphoinositide 3-kinase activation. J Immunol 174: 7172-7178, 2005

40. Faried LS, Faried A, Kanuma T, Aoki H, Sano T, Nakazato T, et al: Expression of an activated mammalian target of rapamycin in adenocarcinoma of the cervix: A potential biomarker and molecular target therapy. Mol Carcinog 47: 446457, 2008

41. Faried LS, Faried A, Kanuma T, et al: Inhibition of the mammalian target of rapamycin (mTOR) by rapamycin increases chemosensitivity of CaSki cells to paclitaxel. Eur J Cancer 42: 934-947, 2006

42. Brown RE, Zhang PL, Lun M, et al: Morphoproteomic and pharmacoproteomic rationale for mTOR effectors as therapeutic targets in head and neck squamous cell carcinoma. Ann Clin Lab Sci 36: 273-282, 2006.

43. Bertelsen BI, Steine SJ, Sandvei R, Molven A and Laerum OD: Molecular analysis of the PI3K-AKT pathway in uterine cervical neoplasia: frequent PIK3CA amplification and AKT phosphorylation. Int J Cancer 118: 1877-1883, 2006.

44. Charette ST and McCance DJ: The E7 protein from human papillomavirus type 16 enhances keratinocyte migration in an Akt-dependent manner. Oncogene 15: 7386-7390, 2007.

45. Menges CW, Baglia LA, Lapoint R and McCance DJ: Human papillomavirus type 16 E7 up-regulates AKT activity through the retinoblastoma protein. Cancer Res 66: 5555-5559, 2006

46. Contreras-Paredes A, De la Cruz-Hernández E, MartínezRamírez I, Dueñas-González A and Lizano M: E6 variants of human papillomavirus 18 differentially modulate the protein kinase $\mathrm{B} /$ phosphatidylinositol 3-kinase (akt/PI3K) signaling pathway. Virology 383: 78-85, 2009. 
47. Plas DR and Thompson CB: Akt-dependent transformation: there is more to growth than just surviving. Oncogene 24: 7435-7442, 2005.

48. Sebolt-Leopold JS and Herrera R: Targeting the mitogenactivated protein kinase cascade to treat cancer. Nat Rev Cancer 4: 937-947, 2004

49. Kiaris $\mathrm{H}$ and Spandidos D: Mutations of ras genes in human tumors. Int J Oncol 7: 413-421, 1995.

50. Zachos G and Spandidos DA: Expression of ras protooncogenes: regulation and implications in the development of human tumors. Crit Rev Oncol Hematol 26: 65-75, 1997

51. Rajalingam K, Schreck R, Rapp UR and Albert S: Ras oncogenes and their downstream targets. Biochim Biophys Acta 1773: 1177-1195, 2007.

52. DiPaolo JA, Woodworth CD, Popescu NC, Notario V and Doniger J: Induction of human cervical squamous cell carcinoma by sequential transfection with humanpapillomavirus 16 DNA and viral Harvey ras. Oncogene 4: 395-399, 1989.

53. Durst M, Gallahan D, Jay G and Rhim JS: Glucocorticoidenhanced neoplastic transformation of human keratinocytes by human papillomavirus type 16 and an activated ras oncogene. Virology 173: 767-771, 1989.

54. Alonio LV, Picconi MA, Dalbert D, et al: Ha-ras oncogene mutation associated to progression of papilomavirus induced lesions of uterine cervix. J Clin Virol 27: 263-269, 2003.

55. Córdova-Alarcón E, Centeno F, Reyes-Esparza J, GarcíaCarrancá A and Garrido E: Effects of HRAS oncogene on cell cycle progression in a cervical cancer-derived cell line. Arch Med Res 36: 311-316, 2005.

56. Landro ME, Dalbert D, Picconi MA, et al: Human papillomavirus and mutated $\mathrm{H}$-ras oncogene in cervical carcinomas and pathological negative pelvic lymph nodes: a retrospective follow-up. J Med Virol 80: 694-701, 2008.

57. Mammas IN, Zafiropoulos A, Koumantakis E, Sifakis S and Spandidos DA: Transcriptional activation of $\mathrm{H}$ - and N-ras oncogenes in human cervical cancer. Gynecol Oncol 92: 941-948, 2004.

58. Karlson J, Borg-Karlson AK, Unelius R, et al: Inhibition of tumor cell growth by monoterpenes in vitro: evidence of a Rasindependent mechanism of action. Anticancer Drugs 7: 422-429, 1996.

59. Sklar MD: The ras oncogenese increase the intrinsic resistance of NIH 3T3 cells to ionizing radiation. Science 239: 645-647, 1988.

60. Van Cutsem E, van de Velde H, Karasek P, et al: Phase III trial of gemcitabine plus tipifarnib compared with gemcitabine plus placebo in advanced pancreatic cancer. J Clin Oncol 22: 1430-1438, 2004.

61. Zhu K, Hamilton AD and Sebti SM: Farnesyltransferase inhibitors as anticancer agents: current status. Curr Opin Investig Drugs 4: 1428-1435, 2003

62. Dammann R, Li C, Yoon JH, Chin PL, Bates S and Pfeifer GP: Epigenetic inactivation of a RAS association domain family protein from the lung tumour suppressor locus $3 \mathrm{p} 21.3$. Nat Genet 25: 315-319, 2000

63. Vos MD, Ellis CA, Bell A, Birrer MJ and Clark GJ: Ras uses the novel tumor suppressor RASSF1 as an effector to mediate apoptosis. J Biol Chem 275: 35669-35672, 2000.

64. Cohen Y, Singer G, Lavie O, Dong SM, Beller U and Sidransky D: The RASSF1A tumor suppressor gene is commonly inactivated in adenocarcinoma of the uterine cervix. Clin Cancer Res 9: 2981-2984, 2003.

65. Kuzmin I, Liu L, Dammann R, et al: Inactivation of RAS association domain family $1 \mathrm{~A}$ gene in cervical carcinomas and the role of human papillomavirus infection. Cancer Res 63: 1888-1893, 2003.

66. Kang S, Kim HS, Seo SS, Park SY, Sidransky D and Dong SM: Inverse correlation between RASSF1A hypermethylation, KRAS and BRAF mutations in cervical adenocarcinoma. Gynecol Oncol 105: 662-666, 2007

67. De Castro Junior G, Puglisi F, de Azambuja E, El Saghir NS and Awada A: Angiogenesis and cancer: a cross-talk between basic science and clinical trials (the 'do ut des' paradigm). Crit Rev Oncol Hematol 59: 40-50, 2006.
68. Folkman J: Seminars in Medicine of the Beth Israel Hospital, Boston Clinical applications of research on angiogenesis. N Engl J Med 333: 1757-1763, 1995.

69. Brat DJ, Kaur B and VanMeir EG. Genetic modulation of hypoxia induced gene Expression and angiogenesis: relevance to brain tumors. Front Biosci 8: 100-116, 2003.

70. Escudier B, Eisen T, Stadler WM, et al: Sorafenib in advanced clear-cell renal-cell carcinoma. N Engl J Med 356: 125-134, 2007.

71. Hurwitz H, Fehrenbacher L, Novotny W, et al: Bevacizumab plus irinotecan, fluorouracil, and leucovorin for metastatic colorectal cancer. N Engl J Med 350: 2335-2342, 2004

72. Imamura T, Kikuchi H, Herraiz MT, et al: HIF-1alpha and HIF-2alpha have divergent roles in colon cancer. Int J Cancer 124: 763-771, 2009

73. Walboomers JMM, Jacobs MV, Manos MM, Bosh FX, Kummer JA, Shah KV, et al: Human papillomavirus is a necessary cause of invasive cervical cancer worldwide. J Pathol 189: 12-19, 1999.

74. Chen W, Li F, Mead L, White H, Walker J, Ingram DA, et al: Human papillomavirus causes an angiogenic switch in keratinocytes which is sufficient to alter endothelial cell behavior. Virology 367: 168-174, 2007.

75. Gaffney DK, Haslam D, Tsodikov A, Hammond E, Seaman J, Holden J, et al: Epidermal growth factor receptor (EGFR) and vascular endothelial growth factor (VEGF) negatively affect overall survival in carcinoma of the cervix treated with radiotherapy. Int J Radiat Oncol Biol Phys 56: 922-928, 2003.

76. Monk BJ, Sill MW, Burger RA, Gray HJ, Buekers TE and Roman LD: Phase II trial of bevacizumab in the treatment of persistent or recurrent squamous cell carcinoma of the cervix: a gynecologic oncology group study. J Clin Oncol 27: 1069-1074, 2009.

77. López-Ocejo O, Viloria-Petit A, Bequet-Romero M Mukhopadhyay D, Rak J and Kerbel RS: Oncogenes and tumor angiogenesis: the HPV-16 E6 oncoprotein activates the vascular endothelial growth factor (VEGF) gene promoter in a p53 independent manner. Oncogene 19: 4611-4620, 2000.

78. Coussens LM, Hanahan D and Arbeit JM: Genetic predisposition and parameters of malignant progression in K14-HPV16 transgenic mice. Am J Pathol 149: 1899-1917, 1996.

79. Smith-McCune KK and Weidner N: Demonstration and characterization of the angiogenic properties of cervical dysplasia. Cancer Res 54: 800-804, 1994.

80. Bequet-Romero $\mathrm{M}$ and López-Ocejo O: Angiogenesis modulators expression in culture cell lines positives for HPV-16 oncoproteins. Biochem Biophys Res Commun 277: 55-61, 2000.

81. Wright JD, Viviano D, Powell MA, Gibb RK, Mutch DG, Grigsby PW, et al: Bevacizumab combination therapy in heavily pretreated, recurrent cervical cancer. Gynecol Oncol 103: 489-493, 2006.

82. Bachtiary B, Schindl M, Potter R, Dreier B, Knocke TH, Hainfellner JA, et al: Overexpression of hypoxia-inducible factor 1 alpha indicates diminished response to radiotherapy and unfavorable prognosis in patients receiving radical radiotherapy for cervical cancer. Clin Cancer Res 9: 2234-2240, 2003.

83. Nakamura M, Bodily JM, Beglin M, Kyo S, Inoue $\mathbf{M}$ and Laimins LA: Hypoxia-specific stabilization of HIF-1alpha by human papillomaviruses. Virology 387: 442-448, 2009.

84. Shinghi AD and Westra WH: Comparison of human papillomavirus in situ hybridization and p16 immunohistochemistry in the detection of human papillomavirus-associated head and neck cancer based on a prospective clinical experience. Cancer 116 : 2166-2173, 2010.

85. Ang KK, Harris J, Wheeler R, et al: Human papillomavirus and survival of patients with oropharyngeal cancer. N Engl J Med 363: 24-35, 2010 . 